\title{
La importancia de compartir los datos
}

\author{
The importance of data sharing
}

Ya hace tiempo se ha comprendido que la investigación científica es el camino para dar respuesta adecuada a los problemas de salud de la población. Esto incluye todo el amplio espectro de diferentes escenarios donde la salud humana es valorada: diagnóstico, tratamiento, calidad, educación profesional, etc.

Pero, además de este primordial aporte, la investigación colabora en desarrollar una sana actitud crítica, que no sólo ayudará a tomar mejores decisiones para nuestros pacientes, sino que también influirá positivamente en otros aspectos de nuestra vida.

Llamativamente, los reportes de una actividad tan importante son, en definitiva, una "declaración jurada". El valor de esta "declaración jurada" sólo se basa en la confianza de la comunidad científica en el investigador.

Si por un momento pensamos en el último artículo de investigación original que hemos leído, seguramente recordaremos que el autor nos muestra en sus resultados que evaluó a una determinada cantidad de pacientes que luego de cierta exposición respondieron de determinada manera. Debemos reconocer que no hemos visto a ninguno de los pacientes que este investigador menciona, y mucho menos hemos podido evaluar cómo se comportaron luego de la exposición investigada. Simplemente confiamos en lo que el autor nos dice.

A pesar del altruismo con el que, generalmente, se conduce la investigación, no hay duda que pueden existir fuertes conflictos de interés, y no puede soslayarse completamente la posibilidad de sesgos inaceptables, o aun fraude. La comunidad científica, a lo largo del tiempo, ha desarrollado mecanismos para prevenir, o al menos disminuir, la posibilidad de que esto ocurra.

La primera defensa de la integridad de las investigaciones está dada por los Comités de Ética en Investigación. El requisito de que las investigaciones sean previamente evaluadas y aprobadas por estos comités garantizan, además de la protección de los sujetos, un manejo adecuado de los conflictos de interés que se presenten.

Las publicaciones científicas han agregado la necesidad del registro previo de los proyectos de investigación. Este requisito, inicialmente diseñado para evitar el sesgo de que sólo se publicaran resultados positivos en investigación farmacológica, pronto se extendió a otro tipo de estudios y garantiza, en líneas generales, la transparencia de la investigación.

Luego de estas primeras barreras se encuentra la revisión por pares. Aunque muy criticado y aun reconociendo que no es un método perfecto, este es todavía el mejor mecanismo con el que contamos para seleccionar qué investigaciones respaldar a través de la publicación científica.

Más recientemente se ha agregado una nueva línea defensiva para mejorar la transparencia en investigación científica. Se trata del intercambio de datos o uso de datos compartidos, más conocido por su nombre en inglés, "data sharing".

El data sharing es la práctica de poner a disposición de otros investigadores los datos no procesados ("crudos") de una investigación. El data sharing incrementa la transparencia de las investigaciones al permitir la confirmación de la interpretación de los resultados. Pero también maximiza la utilidad de los datos, al permitir que sean utilizados en otras investigaciones. ${ }^{1}$

Como en todo componente de una investigación, este punto también debe ser contemplado en el protocolo que la guía. Por un lado, se debe considerar que para poder ser compartidos los datos deben contar con ciertas características conocidas como principios FAIR (acrónimo de Findable, Accessible, Interoperable, Reusable) que incluye ser "encontrables", "accesibles", "interoperables" y "reutilizables". ${ }^{2}$ Por otro lado, el plan de compartir datos también debe considerar qué datos se compartirán, quién podrá acceder a ellos, dónde se guardarán, cuándo serán compartidos y cómo se accederá a los datos. Estos mismo elementos son, en general, los que se consignan en la declaración de política de compartir datos que las publicaciones exigen con la remisión del manuscrito. El Comité Internacional de Editores de Revistas Médicas (ICMJ) brinda ejemplos de cómo suscribir esta declaración. ${ }^{3}$

Aunque la iniciativa de data sharing fue concebida inicialmente para la investigación clínica, ${ }^{4}$ actualmente se ha visto la conveniencia de extender la práctica a todas las investigaciones, 
incluso la observacionales. ${ }^{5}$

Como toda iniciativa nueva, el data sharing también ha generado algunas controversias. Existe una preocupación relacionada con la confidencialidad y la privacidad de los participantes en investigaciones. También existe el riesgo que el análisis secundario de los datos, sacados fuera del contexto para el que fueron recogidos, lleven más fácilmente a resultados poco confiables en las investigaciones secundarias. Finalmente, también genera inquietud que la actividad de los investigadores "primarios" se vea amenazada por aquellos que usan secundariamente sus datos y puedan alcanzar más visibilidad y prestigio. ${ }^{6}$ Aunque los puntos antes mencionados aún son tema de debate, los primeros pueden ser discutidos con el Comité de Ética, mientras que el último puede ser zanjado con el reconocimiento apropiado al investigador primario por parte de los usuarios de datos compartidos.

Las publicaciones científicas tienen una responsabilidad fundamental en velar por la integridad de las investigaciones cuyos datos publican. La exigencia de que las investigaciones hayan sido aprobadas por un comité de ética y registradas en un registro público previo a su desarrollo, el compromiso de compartir los datos y la revisión por pares son elementos importantes para respaldar este compromiso.

Archivos Argentinos de Pediatría adhiere a estas prácticas en su esfuerzo por ofrecer evidencia científica de la mejor calidad y con la mayor transparencia. http: / / dx.doi.org/10.5546/ aap.2022.74

Texto completo en inglés:

http:/ / dx.doi.org/10.5546/ aap.2022.eng.74

Cómo citar: Ferrero F. La importancia de compartir los datos. Arch Argent Pediatr 2022;120(2):74-75.

\section{REFERENCIAS}

1. Sixto-Costoya A, Aleixandre-Benavent R, Vidal-Infer A, Lucas-Domínguez R, Castelló-Cogollos L. Data sharing: qué son y cómo se pueden compartir los datos de investigación. Manual de recomendación para gestores de la información. Sociedad Española de Documentación e Información Científica, 2019. [Consulta: 24 denoviembre de 2021]. Disponibleen: https: / / www.sedic.es / wp-content / uploads / 2020/01/Data_Sharing-DEF.pdf

2. Wilkinson MD, Dumontier M, Aalbersberg IJ, Appleton G, et al. The FAIR Guiding Principles for scientific data management and stewardship. Sci Data. 2016;3:160018.

3. International Committee of Medical Journal Editors. Recommendations. Data sharing. [Consulta: 24 de noviembre de 2021]. Disponible en: http:/ / www.icmje. org/recommendations/browse/ publishing-and-editorialissues / clinical-trial-registration.html\#: :text=Data\%20 sharing $\% 20$ statements $\% 20$ must $\% 20$ indicate,(e.g. $\% 2 \mathrm{C} \% 20$ study $\% 20$ protocol $\% 2 \mathrm{C} \% 20$ statistical

4. Committee on Strategies for ResponsibleSharing of Clinical Trial Data; Board on Health Sciences Policy; Institute of Medicine. Sharing Clinical Trial Data: Maximizing Benefits, Minimizing Risk. Washington (DC): National Academies Press (US); April 20, 2015.

5. Ewers M, Ioannidis JPA, Plesnila N. Access to data from clinical trials in the COVID-19 crisis: open, flexible, and time-sensitive. J Clin Epidemiol. 2021;130:143-6.

6. Bull S, Cheah PY, Denny S, Jao I, et al. Best practices for ethical sharing of individual-level health research data from low- and middle-income settings. J Empir Res Hum Res Ethics. 2015;10(3):302-13.
Fernando Ferrero (ID)

Editor 